Michèl Gehrke

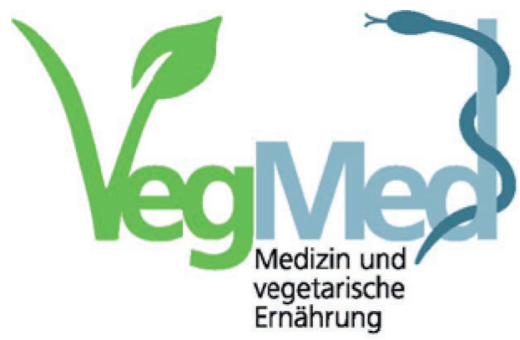

Abb. 1. VegMed - erster wissenschaftlicher Ärztekongress für vegetarische Ernährung im deutschsprachigen Raum.

Veganer: Weniger Übergewicht und geringeres Diabetesrisiko

Dr. Markus Keller vom Institut für alternative und nachhaltige Ernährung (IFANE) referierte über in Studien beobachtete Vorteile einer vegetarischen bzw. veganen Ernährung. Dabei führte er unter den prospektiven Kohortenstudien vor allem die zweite "Adventist Health Study» [1] an. Diese zeigte bei den untersuchten Veganern einen um etwa fünf Einheiten niedrigeren Body-Mass-Index (BMI) als bei Fleischkonsumenten. Eine Zwischenstellung nehmen die Vegetarier, die auch Ei- und Milchprodukte zu sich nehmen (Ovo-LaktoVegetarier), Fischesser und SeltenFleischesser ein. Das Übergewichtsrisiko steigt demnach mit dem Konsum von tierischen Produkten, womit auch eine erhöhte Prävalenz für Diabetes mellitus Typ 2 einhergeht: Diese liegt bei 2,9\% bei Veganern im Vergleich zu 7,6\% bei Fleischkonsumenten. Zwar relativiert sich diese Diskrepanz leicht, sobald neben dem BMI auch andere Faktoren wie die durchschnittliche Schlafdauer, der Grad an regelmässiger Bewegung usw. berücksichtigt werden, doch selbst nach Adjustierung ist das Diabetes-

\title{
Årztekongress VegMed 2012: Vegetarische Ernährung und Medizin - solide Evidenz, aber bessere Umsetzung in die Praxis nötig
}

\begin{abstract}
Auf der «VegMed 2012» (Abb. 1, 2), dem ersten wissenschaftlichen Ärztekongress für vegetarische Ernährung im deutschsprachigen Raum, haben Ärzte und Ernährungswissenschaftler am 9. Dezember 2012 in Berlin die themenbezogene Datenlage zusammengetragen und über Zukunftsperspektiven diskutiert.
\end{abstract}

risiko der Veganer nur etwa halb so gross wie das der Fleischesser.

\section{Günstigere Nährstoffzusammen- setzung positiv für das Herz- Kreislauf-System}

Dies trifft auch für kardiovaskuläre Erkrankungen zu, wie Prof. Andreas Michalsen, Inhaber der Stiftungsprofessur für klinische Naturheilkunde der Charité im Immanuel Krankenhaus Berlin, verdeutlichte. Von den grossen epidemiologischen Studien zum Thema griff er etwa die 7-Länder-Studie [2] heraus, nach der in Kreta und Japan das Risiko für ein metabolisches Syndrom im Studienvergleich am geringsten ist - Regionen, in denen sich die Menschen traditionell überwiegend vegetarisch ernähren. Der niedrigere BMI sei dabei nicht allein entscheidend, sondern auch die Tatsache, dass Vegetarier ernährungsbedingt weniger gesättigte Fette und Cholesterin zu sich nehmen, dafür aber einen höheren Konsum von Ballaststoffen, Antioxidantien und anderen sekundären Pflanzenstoffen aufweisen. Ein starker Fleischkonsum bringt eine gesteigerte Eisenaufnahme mit sich, die wiederum mit einem erhöhten Risiko für Herzinfarkte, Bluthochdruck und letztlich einer gesteigerten Mortalität einhergeht [3]. Pflanzenbetonte Ernährung führt deshalb tendenziell $\mathrm{zu}$ besseren Blutfettwerten, einem güns- tigeren Blutzuckerspiegel und einem niedrigeren Blutdruck [4]. Tatsächlich gibt es Untersuchungen, bei denen eine überwiegend vegetarische Diät mit viel Obst und Gemüse, vielen Vollkornprodukten und Nüssen, aber wenig gesättigten Fetten und ohne rotes Fleisch und Zucker den Blutdruck bei Hypertonikern stärker senkt als eine medikamentöse Therapie ohne diätetische Massnahmen $[5,6]$.

Darüber hinaus hebt unter anderem die klinische Studie von McEvoy et al. [7] die Vorzüge einer mediterranen, überwiegend vegetarischen Ernährung bei Demenz, rheumatoider Arthritis und Fibromyalgiesyndrom, Divertikulose, Katarakt, Osteoporose sowie Nieren- und Gallensteinen hervor. Dass eine vorwiegend pflanzliche Ernährung Vorzüge aufweist, ist evident - laut Andreas Michalsen ist die Frage derzeit eher: Welche Form der vegetarischen Ernährung wirkt nachhaltiger und was bedeutet das für die klinische Praxis?

Eine Antwort lieferte Prof. Claus Leitzmann, ehemaliger Leiter des Giessener Instituts für Ernährungswissenschaft. Er sprach sich für eine vollwertige vegetarische Ernährung mit einem hohen Anteil an pflanzlicher Kost aus, da diese die optimale Mischung der vom Menschen benötigten Nährstoffe enthält. Sogar eine ausschliesslich pflanzliche Ernährung

\section{KARGER}

Fax +4976145207 14
(๑) 2013 S. Karger GmbH, Freiburg

Accessible online at: www.karger.com/szg
Michèl Gehrke

Pressestelle Karl und Veronica Carstens-Stiftung

Am Deimelsberg 36, 45276 Essen, Deutschland

M.Gehrke@carstens-stiftung.de

www.carstens-stiftung.de 


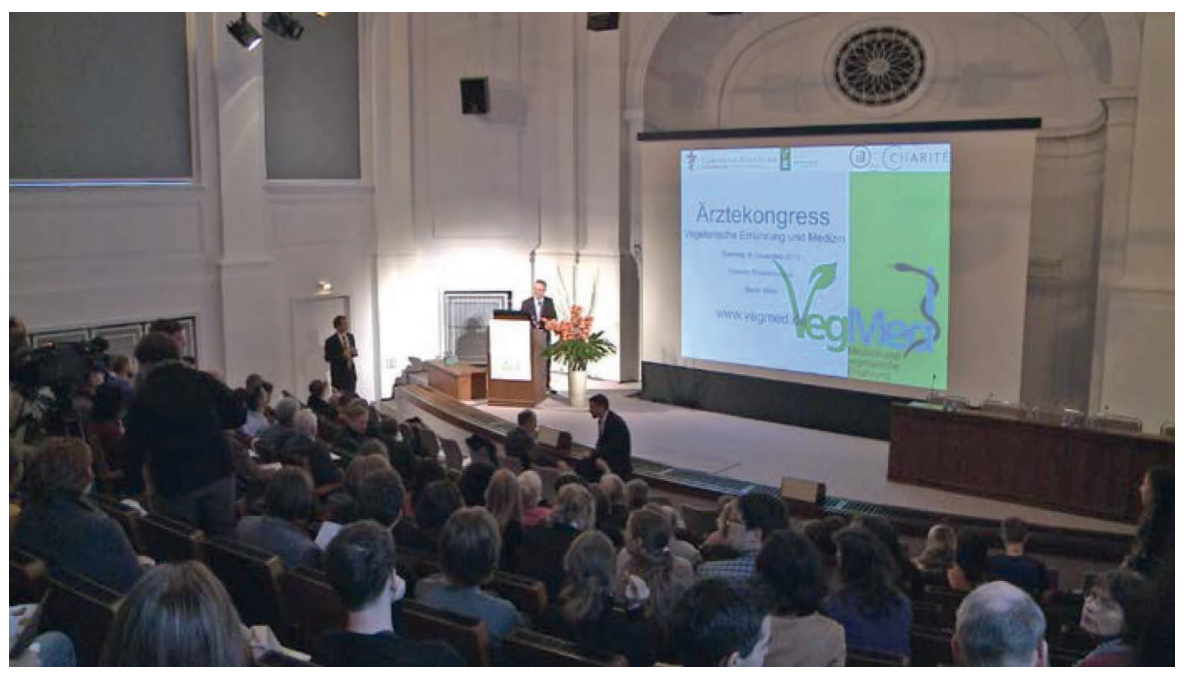

Abb. 2. VegMed 2012 - mit 250 Teilnehmern ein voller Erfolg.

ist bedarfsgerecht und gesundheitlich sinnvoll, solange die Kost vielfältig und qualitativ hochwertig ist und auf eine ausreichende Vitamin-B12-Zufuhr geachtet wird.

\section{Diskrepanz zwischen Evidenz und Praxis}

Von der Ärzteschaft werden die Vorzüge dieser Ernährungsformen bislang kaum wahrgenommen oder umgesetzt, so das einhellige Credo des Plenums aus Ärzten, Medizinstudierenden und Angehörigen ernährungswissenschaftlicher Fachberufe. Die Frage nach den Ursachen hierfür sowie entsprechende Lösungsvorschläge wurden in Podiumsdiskussionen mit den Referenten intensiv diskutiert. Ein möglicher Faktor ist, dass das Thema Ernährung im Medizinstudium kaum behandelt wird und deshalb nur wenigen Ärzten präsent ist. Regelmässige Fort- und Weiterbildungen könnten hier Abhilfe schaffen. Auf der anderen Seite berichten Ernährungstherapeuten immer wieder davon, dass Patienten sich nur schwer vom Fleischverzicht überzeugen lassen. Als Alternative zu verbotsbetonter Vermittlung wurde vorgeschlagen, Patienten stattdessen Verhaltensänderungen über positive Formulierungen nahezubringen und mit ressourcenorientierter Herangehensweise die kulinarischen Vorzüge und die Viel- falt der vegetarischen Küche zu veranschaulichen. Als strukturelles Defizit konnte schliesslich die mangelnde Vernetzung der Ärzte mit Ernährungsberatern oder Diätassistenten identifiziert werden. Viel zu selten verweisen die Mediziner ihre Patienten an die entsprechenden Fachleute, obwohl ausreichend geschultes Personal vorhanden ist und die Kassen diese Leistungen übernehmen.

Das Resümee von Andreas Michalsen: $" \mathrm{Ob}$ die vegetarische Ernährung mehr Einzug in die Medizin erhalten wird, ist weniger eine Frage der Evidenz als vielmehr eine Frage von Kultur, Konsumgewohnheiten und Nachhaltigkeit.»

\section{VegMed 2012 ein voller Erfolg - Videos der Vorträge online}

Mit 250 Teilnehmern zeigten sich die Veranstalter hochzufrieden. Die Konferenz war trotz Bereitstellung zusätzlicher Räumlichkeiten schon Wochen vorher ausgebucht; das Publikum äusserte sich in der Evaluation der Veranstaltung überaus positiv. Für Interessierte, die an der Veranstaltung nicht teilnehmen konnten, sind die Vorträge der Referenten als Video kostenlos und ohne Registrierung online unter www.vegmed.org abrufbar.

\section{Ausblick: VegMed 2013}

Aufgrund der grossen Nachfrage wird die Konferenz in diesem Jahr mit neuem Programm und in grösserem Rahmen erneut ausgerichtet. «VegMed 2013» wird am 17. November 2013 im Kaiserin-Friedrich-Haus in Berlin stattfinden. Dabei wird auch in diesem Jahr wieder für eine hochwertige und schmackhafte vegetarische Verpflegung gesorgt. Details zu Anmeldung und Programm werden in Kürze auf www.vegmed.org zur Verfügung gestellt.

VegMed wird von der Karl und Veronica Carstens-Stiftung finanziert und zusammen mit der Abteilung für Naturheilkunde der Charité am Immanuel Krankenhaus und dem Vegetarierbund Deutschland (VEBU) organisiert. Die Tagung wird sich als voraussichtlich Continual-MedicalEducation(CME)-zertifizierte Fortbildung insbesondere an Ärzte richten. Diätassistenten, Ernährungswissenschaftler und Biologen können jedoch ebenfalls teilnehmen.

\section{Kontakt}

Dr. Christian Kessler

Forschungskoordination

Charité Hochschulambulanz für Naturheilkunde am Immanuel-Krankenhaus Berlin-Wannsee

Königstrasse 63, 14109 Berlin,

Deutschland

C.Kessler@immanuel.de www.naturheilkunde.immanuel.de

Elmar Stapelfeldt

Wissenschaftlicher Mitarbeiter Charité Hochschulambulanz für Naturheilkunde am Immanuel-Krankenhaus Berlin-Wannsee

Königstrasse 63, 14109 Berlin,

Deutschland

E.Stapelfeldt@immanuel.de

Sebastian Zösch

Geschäftsführer Vegetarierbund

Deutschland e.V. (VEBU)

Glatzer Strasse 5, 10247 Berlin,

Deutschland

Sebastian.Zoesch@vebu.de www.vebu.de 
Michèl Gehrke

Pressestelle Karl und Veronica Carstens-

Stiftung

Am Deimelsberg 36, 45276 Essen,

Deutschland

M.Gehrke@carstens-stiftung.de

www.carstens-stiftung.de

\section{Literatur}

-1 Tonstad S, Butler T, Yan R, Fraser GE: Type of vegetarian diet, body weight, and prevalence of type 2 diabetes. Diabetes Care 2009;32:791-796
2 Keys A: Seven Countries: A Multivariate Analysis of Death and Coronary Heart Disease. Cambridge, Harvard University Press, 1980.

3 Rohrmann S, Overvad K, Bueno-de-Mesquita $\mathrm{HB}$, et al: Meat consumption and mortality - results from the European Prospective Investigation into Cancer and Nutrition. BMC Med 2013;11:63.

-4 Rizzo NS, Sabaté J, Jaceldo-Siegl K, Fraser GE: Vegetarian dietary patterns are associated with a lower risk of metabolic syndrome: the adventist health study 2 . Diabetes Care 2011;34:1225-1227.

Dehghan M, Mente A, Teo KK, Gao P, Sleight P, Dagenais G, Avezum A, Probstfield JL, Dans T, Yusuf S: Relationship between healthy diet and risk of cardiovascular disease among patients on drug therapies for secondary prevention: a prospective cohort study of 31,546 high-risk individuals from 40 countries. Circulation 2012;126:27052712.

6 Appel LJ, Moore TJ, Obarzanek E, Vollmer WM, Svetkey LP, Sacks FM, Bray GA, Vogt TM, Cutler JA, Windhauser MM, Lin PH, Karanja N: A clinical trial of the effects of dietary patterns on blood pressure. DASH Collaborative Research Group. N Engl J Med 1997;336:1117-1124.

7 McEvoy CT, Temple N, Woodside JV: Vegetarian diets, low-meat diets and health: a review. Public Health Nutr 2012;15:22872294. 